\title{
Parking Assistance Display WITH ESTIMATED PARKING SPACE USING STEREO VISION
}

\author{
Chi-Cheng Cheng, Chi-Cheng Lee and Jyun-Han Huang \\ Department of Mechanical and Electro-Mechanical Engineering, \\ National Sun Yat-Sen University, Kaohsiung, Taiwan, R.O.C.
}

\begin{abstract}
Inexperienced drivers always suffer from limited spatial information coming from side and review mirrors to complete parking tasks. The major obstacle is that they cannot easily estimate relative position of the parking space with respect to their vehicles. Therefore, this paper aims to develop a parking assistance display system that can continuously provide the top view of both the vehicle and the parking space for drivers. The system applies two wide-angle cameras mounted at the rear of the vehicle. In order to search for two farther corners of the parking space with efficiency, the FAST corner detection technique is employed. Three dimensional spatial coordinates of those corners can therefore be determined by the stereo vision framework. As a result, the position of the parking space relative to the vehicle can be estimated. To verify the effectiveness of the proposed parking assistance display, actual parking experiments with a golf cart were conducted. Experimental results demonstrate the parking tasks can be successfully accomplished with the help from the presented assistance display.
\end{abstract}

\section{KEYWORDS}

Parking Assistance Display, Stereo Vision, Corner Detection, Parking Space Estimation

\section{INTRODUCTION}

Parking is always a great challenge for drivers and strongly requires not only matured driving skills, but also accumulation of parking experience. Traditional parking assistance device consists of only the review and side mirrors. Based on the images from those mirrors, human drivers can approximately estimate environment including the parking space around the vehicle. Because of highly development of microelectronics, most parking information for drivers now comes from rear parking sensors, which give warning signal by detecting rear distance and is quite useful for parking tasks including parallel parking and garage parking. Recently, the active parking assist relying on supersonic sensor technology becomes more and more popular and the parking task can almost be accomplished without the help from drivers [1]. However, unexpected road conditions may occur anytime, human drivers still need to play the role of a supervisor especially when manual intervention on the automatic system is necessary.

The difficulty of parking stems from a number of inevitable facts. The major constraint is that the driver sits inside the vehicle's body and the clues about the environment can only be achieved by putting together pieces of image information gathered from review and side mirrors as well as visual impression from looking out of the window. Although each visual image provides partial three-dimensional environmental information, it is difficult for drivers to realize what the actual environment is based on those discontinuous and incomplete visual data.

David C. Wyld et al. (Eds): SPTM, IPPR, AIS, CSIT, DaMi, AMLA - 2020

pp. 35-44, 2020. CS \& IT - CSCP 2020

DOI: $10.5121 /$ csit.2020.100604 
Due to rapid progress on imaging technology, electronics fabrication, and computational capability, machine vision has been widely applied to many manufacturing areas including the automotive industry. Currently, the category of vision-based studies on automobiles probably is one of the popular research fields regarding applications of machine vision. For the purpose of preventing difficulty from parking in dark indoor site, a parking space recognition method with the light stripe projection approach was presented in [2]. To automate the target position selection of an automatic parking assist system, a parking-slot-markings recognition algorithm using two seed-points, end-points of two line segments separating the target parking slot from adjacent ones, was proposed to reduce the search range and memory requirement [3].Incorporating an around view monitor (AVM) system with a hierarchical tree structure approach, various parking slot markings can be almost fully automatic recognized [4]. Continuing from previous research, in [5], a vacant parking slot detection and tracking system using the sensor fusion technology combing an AVM system and an ultrasonic sensor was developed in 2014.

In order to offer extensive visual information about the surroundings of the vehicle, a composite top-down view of $360^{\circ}$ of the vehicle was synthesized in [6] by combining images taken from four to six wide-angle cameras mounted around the vehicle. Similarly, an automatic parking framework based on a bird's eye vision system using four on-board fisheye cameras around the vehicle with the double circular trajectory planning and a preview control strategy was also presented [7]. In [8], a portable real-time informational bird's eye view system for the advanced driver assistance system was actually implemented. Furthermore, a parking assistance system with the depth map of the observed environment computed from dense motion-stereo was proposed to allow drivers to be able to visualize the environment around the host vehicle [9]. Compared with the ultrasound approach, the proposed approach demonstrated better accuracy and reliability in terms of both quantitative and qualitative results. Although vision-based approaches for automatic parking tasks in the field of intelligent vehicles gain interests from both academic institutions and automotive industry, there was no public benchmark dataset available for evaluation of parking-slot detection algorithms. Fortunately, a large-scale parking-slot image database comprising 8600 surround-view images collected from indoor and outdoor parking sites was established [10]. Besides, at the same time, a learning-based parking-slot detection approach was also proposed.

If estimated position of the park space relative to the vehicle can be displayed for the drivers using extra cameras, it would be helpful for drivers to conduct parking tasks since human drivers are aware of where the parking space is located. The stereo vision system is able to offer the depth information of objects in three-dimensional scene and has the cost effective advantage than other vision devices such as 360-degree panorama cameras. Besides, although the 360-degree panorama cameras are capable of delivering whole visual information around the vehicle, images for objects in the environment always suffer from severe distortion and poor image quality. Consequently, a binocular vision system installed on the back of the vehicle will be applied for position estimation of the parking space with respect to the vehicle in this study.

\section{Corner Detection for Parking SPaCe}

In order to provide the position information for the parking space, an effective image processing algorithm for locating the parking space plays a crucial role. The algorithm needs to be computational efficiency to meet the requirement of fast estimation for parking tasks.

The parking space for regular cars has a shape of rectangular with the length of 5 5.7 meters and the width of 2 2.5 meters. The best feature for parking space detection should be its four corners. The FAST (Features from Accelerated Segment Test) method developed by Rosten and 
Drummond in 2006 provides a time efficient algorithm for corner detection [11]. This approach starts with examining intensity difference between 16 adjacent pixels around a mask e.g., $7 \times 7$ over the target point. If the number of pixels that fulfil a given threshold value is greater than a certain number, e.g., 9, then the target point should be considered as a point at the corner. The OpenCV (Open Source Computer Vision Library), an open source computer vision and machine learning software library, offers a number of different masks for high-speed corner detection according to the FAST concept.

Since the stereo cameras are installed on the back of the vehicle, estimation of the parking space will be achieved by examining those two corners of the parking space far away from the vehicle due to the fact that they are easily captured by the cameras. The TYPE_7_12 FAST function, a $5 \times 5$ mask with 7 out of 12 pixels as depicted in Figure 1, was chosen for corner detection in small area. Furthermore, detection sensitivity is improved by applying 5 out of 12 pixels instead of regular 7 out of 12 pixels to prevent from unsuccessful detection for the left corner being an obtuse angle. After the FAST corner detection algorithm, Figure 2 demonstrates pixels for corner candidates.

In order to select appropriate inner corner point among many corner candidates from the FAST corner detection algorithm, three criteria are established. Take the right corner as an example shown in Figure 3. The first criterion is that if the point $\mathrm{P}$ is an inner corner point, the following conditions have to be satisfied.

$$
\mathrm{G}_{3}>\mathrm{G}_{\mathrm{P}} \text { and } \mathrm{G}_{3}>\mathrm{G}_{9} \text {, }
$$

Where $G_{i}$ represents the gray scale of the $i$-th pixel. The second criterion is that the points with significantly different angular style need to be removed. Figure 4 illustrates a right corner described by pixels 69 and 89 in a 112-pixel circular mask. With \pm 4 pixels tolerance, only points within the ranges of 65-73 and 85-93 will be reserved for further inner corner verification. After passing the first two criteria, the remaining pixels are always located around the inner corner. The unique inner corner pixel can be easily obtained with the final criterion of the smallest included angle.

While the driver is performing the parking task, the scene grabbed by the cameras is always different from that taken at previous time instant. In order to enhance computational efficiency for corner detection, the detected inner corner from previous time instant will be applied as the center of ROI (Region of Interest) for searching for inner corners of the parking space at the current time instant. Besides, the angular style solved from criteria 1 and 2 at previous moment will be directly used as the initial condition for the current instant.
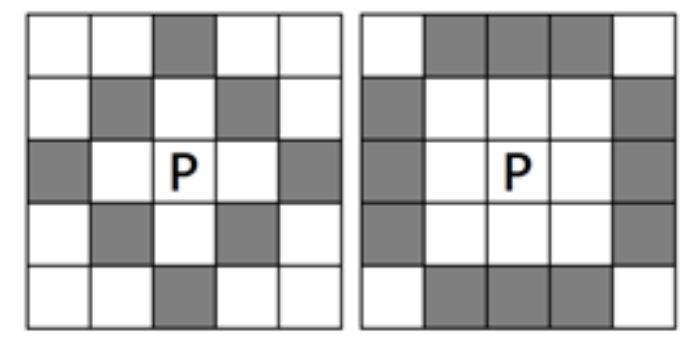

Figure 1. TYPE_5_8 and TYPE_7_12 masks in OpenCV for FAST corner detection 


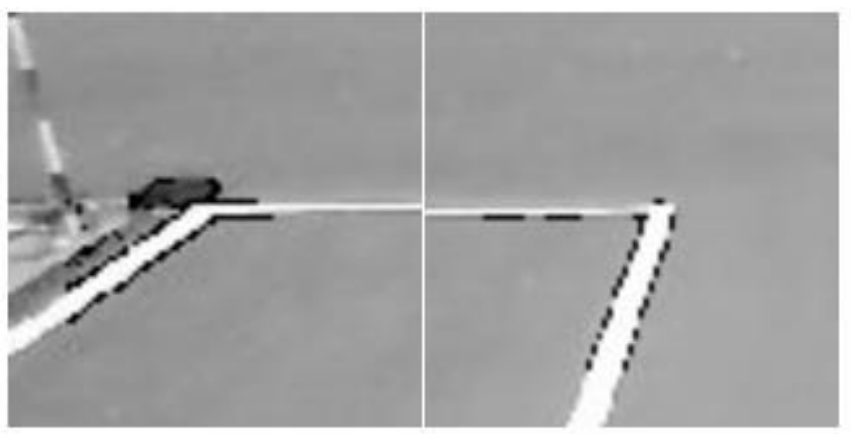

Figure 2. Corner candidates after FAST corner detection algorithm
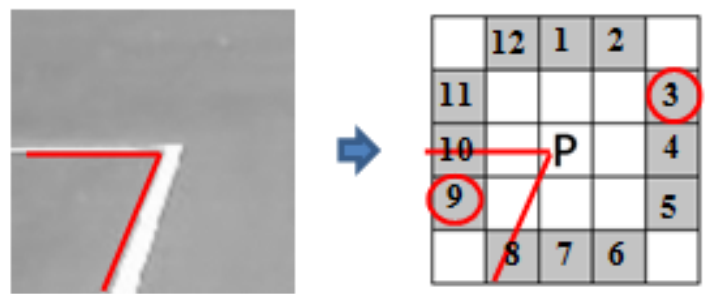

Figure 3. The first criterion for inner corner detection

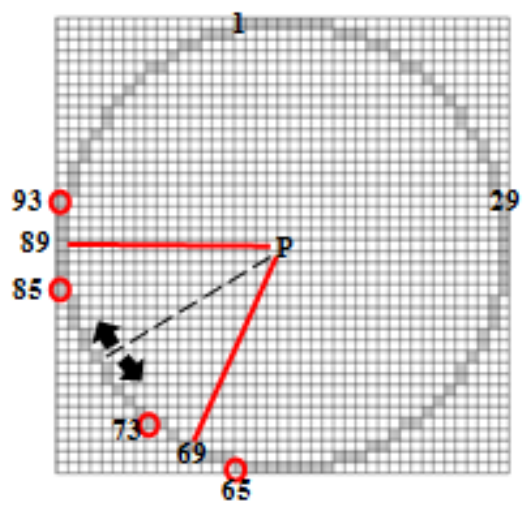

Figure 4.The second criterion for inner corner detection

\section{3. estimation of the Parking Space}

After successful corner detection described in previous section, the three-dimensional coordinates of the parking space will be determined by a binocular vision framework. The binocular vision framework provides two images at the same time and is able to solve depth information of the target point using the clue of disparity defined as the difference between project points on two image planes.

Let two identical cameras be placed laterally with a baseline distance $D$ and their optical axes are parallel with each other as illustrated in Figure 5. Based on the model of the stereo vision, the projection points $\left(u_{R}, v_{R}\right)$ and $\left(u_{L}, v_{L}\right)$ on the right and left image planes respectively can be expressed by 


$$
\begin{aligned}
& u_{R}=\alpha_{R} \frac{x_{R}}{z_{R}}+u_{0 R}, v_{R}=\beta_{R} \frac{y_{R}}{z_{R}}+v_{0 R}, \\
& u_{L}=\alpha_{L} \frac{x_{L}}{z_{L}}+u_{0 L}, v_{L}=\beta_{L} \frac{y_{L}}{z_{L}}+v_{0 L},
\end{aligned}
$$

Where $\alpha_{i}$ and $\beta_{i}$ are products of the focal length and the aspect ratio in horizontal and vertical directions due to non-square pixels, respectively. Besides, $\left(u_{0 R}, v_{0 R}\right)$ and $\left(u_{0 L}, v_{0 L}\right)$ stand for the offsets between the image center and the principal point because of imperfect assembly of image sensors. Those intrinsic camera parameters can be solved by standard calibration procedures.

After a number of mathematical manipulations, the target position $\left(x_{R}, y_{R}, z_{R}\right)$ with respect to the right camera can be obtained as

$$
\begin{gathered}
x_{R}=\frac{\alpha_{L} D\left(u_{R}-u_{0 R}\right)}{-\alpha_{L}\left(u_{R}-u_{0 R}\right)+\alpha_{R}\left(u_{L}-u_{0 L}\right)^{\prime}}, \\
y_{R}=\frac{v_{R}-v_{0 R}}{\beta_{R}} \cdot \frac{\left(\alpha_{R}-\alpha_{L}\right) x_{R}-\alpha_{L} D}{u_{R}-u_{0 R}-\left(u_{L}-u_{0 L}\right)}, \\
z_{R}=\frac{\left(\alpha_{R}-\alpha_{L}\right) x_{R}-\alpha_{L} D}{u_{R}-u_{0 R}-\left(u_{L}-u_{0 L}\right)}
\end{gathered}
$$

Similarly, the same target position but in the left camera coordinate system $\left(x_{L}, y_{L}, z_{L}\right)$ can also be derived as

$$
\begin{gathered}
x_{L}=\frac{\alpha_{R} D\left(u_{L}-u_{0 L}\right)}{-\alpha_{R}\left(u_{L}-u_{0 L}\right)+\alpha_{L}\left(u_{R}-u_{0 R}\right)}, \\
y_{L}=\frac{v_{L}-v_{0 L}}{\beta_{L}} \cdot \frac{\left(\alpha_{L}-\alpha_{R}\right) x_{R}+\alpha_{R} D}{u_{L}-u_{0 L}-\left(u_{R}-u_{0 R}\right)}, \\
z_{L}=\frac{\left(\alpha_{L}-\alpha_{R}\right) x_{R}-\alpha_{R} D}{u_{L}-u_{0 L}-\left(u_{R}-u_{0 R}\right)} .
\end{gathered}
$$

Ideally, $x_{R}=x_{L}-D, y_{R}=y_{L}$, and $z_{R}=z_{L}$. Due to limited image resolution and inevitable noises, those ideal equations cannot be perfectly satisfied. But the target position can still be successfully estimated. For future implementation, a new reference system with its origin at the middle point of origins of both right and left camera coordinate systems is established. Then the target point $\left(x_{C}, y_{C}, z_{C}\right)$ with respect to the new reference system as illustrated in Figure 5 can be simply formulated by

$$
\begin{aligned}
& x_{C}=\frac{x_{R}+x_{L}}{2}, \\
& y_{C}=\frac{y_{R}+y_{L}}{2}, \\
& z_{C}=\frac{z_{R}+z_{L}}{2} .
\end{aligned}
$$


In addition, for the purpose of covering the parking space on the ground, both cameras need to be pointed downwards with an inclination angle $\theta$ with respect to the horizontal level as shown in Figure 6. In order to determine the relative location of the parking space with respect to the vehicle in the global reference frame, the position information extracted by the cameras need to be transformed to a reference system $(X, Y, Z)$ using

$$
\left[\begin{array}{l}
X \\
Y \\
Z
\end{array}\right]=\left[\begin{array}{ccc}
1 & 0 & 0 \\
0 & \cos \theta & \sin \theta \\
0 & -\sin \theta & \cos \theta
\end{array}\right]\left[\begin{array}{l}
x_{C} \\
y_{C} \\
z_{C}
\end{array}\right] .
$$

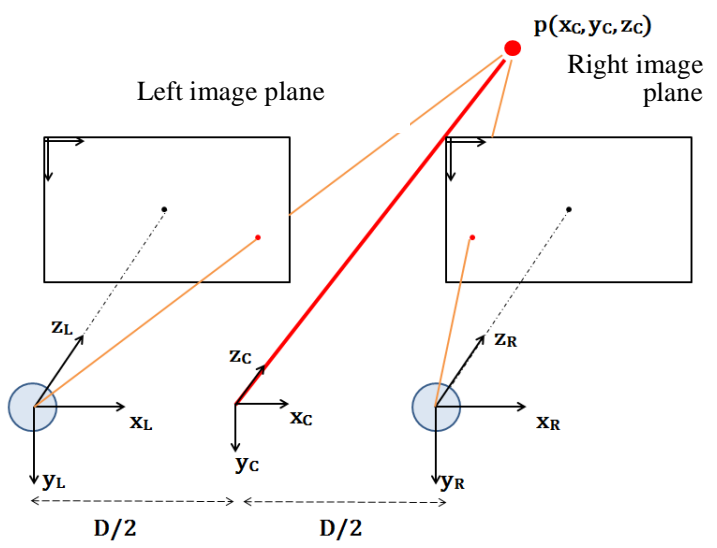

Figure 5. Reference frames for the binocular system

The origin of the reference system $(X, Y, Z)$ coincides with that of the reference system $\left(x_{C}, y_{C}, z_{C}\right)$, but with its $Y$-axis pointing downwards, and both $X$ and $Z$ axes parallel with the ground. It is possible all four corner points may not be captured by the cameras during the parking process especially when the vehicle is close to the parking space. Fortunately, since the dimension of the parking space can be already known, estimation of the parking space can still be accomplished by only two farther corner points.

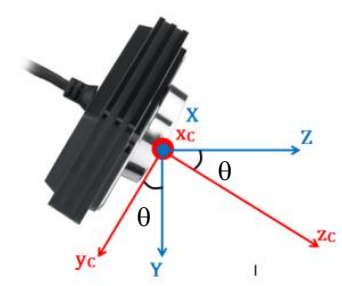

Ground

Figure 6. The camera pointing downwards with an inclination angle

\section{Parking Experiments}

In order to verify performance of the proposed parking assistance display, actual parking experiments with a golf cart were conducted in the campus. The golf cart shown in Figure 7 is equipped with a laptop computer, which generates the parking assistance display to drivers, and a dual-camera system on its back. The golf cart has a length of $240 \mathrm{~cm}$ and a width of $102 \mathrm{~cm}$. The 
cameras are installed on top of the vehicle with a height of $160 \mathrm{~cm}$ and an inclination angle of 30 degrees downwards. The baseline between those two cameras is $20 \mathrm{~cm}$. The camera chosen for experiments is a Widecam 1050 by Genius, which is a CMOS device that owns the advantage of low energy dissipation for portable applications. The camera has a resolution of 640x480 pixels and a wide view angle so that the parking space can be easily captured. All image processing algorithms and the assistance display were performed by a Lenovo t530 laptop computer accompanied with Visual Studio and OpenCV as software development tools. The target parking space is $475 \mathrm{~cm}$ long and $214 \mathrm{~cm}$ wide.

Actual parallel parking experiments were conducted to examine the parking performance with the help from the proposed parking assistance display. For the purpose of preventing the driver from acquiring information around the vehicle using eyes, a black curtain covering the vehicle's body was applied to block the view of the driver. The only clue for performing the parking task was the assistive display shown on the screen of the laptop as depicted in Figure 8. Figure 9 illustrates responses of the relative distance and orientation angle between the vehicle and the parking space, which are defined in Figure 10, for a parking experiment. Figure 11 presents the relationship between the estimated and the actual parking space at 5.8 second. Apparently, position error existed due to imperfect estimation of the location of the parking space. The deviation mainly came from the error caused by inaccurate depth estimation. Furthermore, during parking experiments, the driver seemed being disturbed by inconsistent images of the parking space. Although the location of the parking space could not be exactly estimated, the proposed parking assistance display still provided important information regarding relative position of the vehicle with respect to the parking space. In addition, the estimation accuracy improved when the vehicle got closed to the parking space. As a result, the driver was able to accomplish the parking task just relying on the parking assistance display proposed by this research.

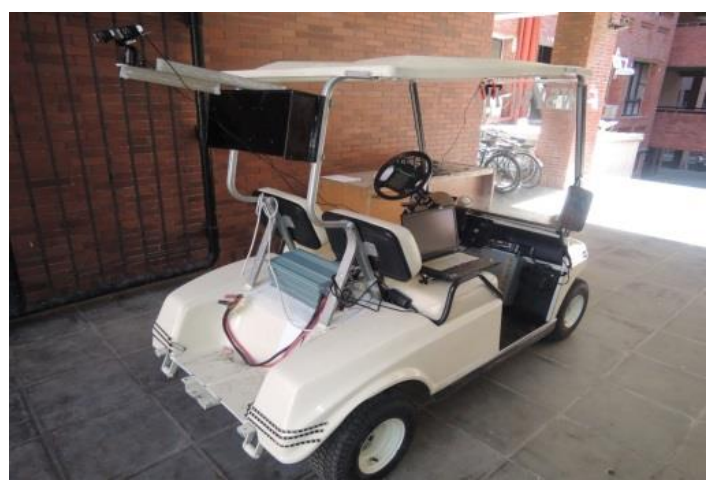

Figure 7. The golf cart with stereo vision for actual parking experiments

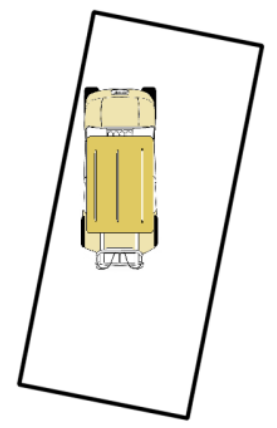

Figure 8. The parking assistance display shown on the screen 

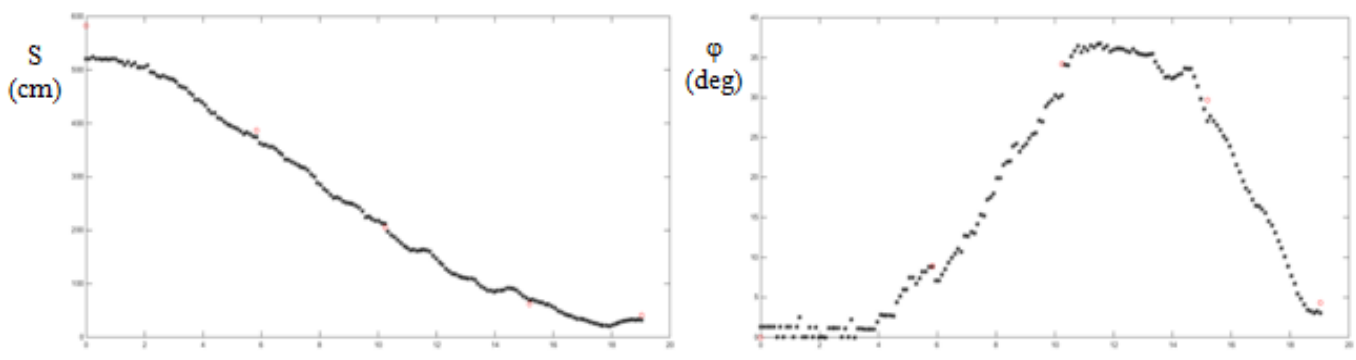

Figure 9. Distance and orientation responses of a parallel parking experiment
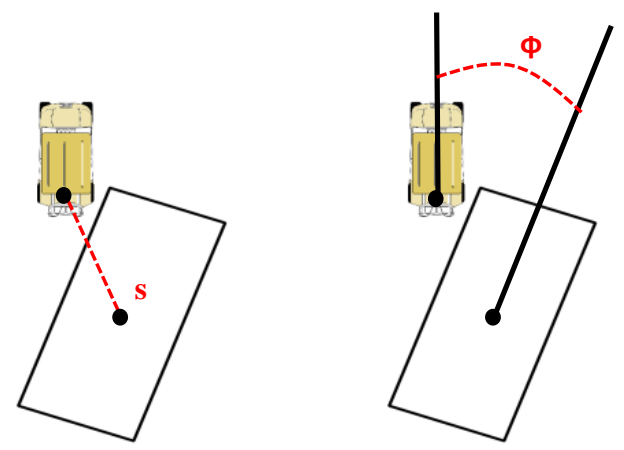

Figure 10. Illustrative distance and orientation for parking experiments

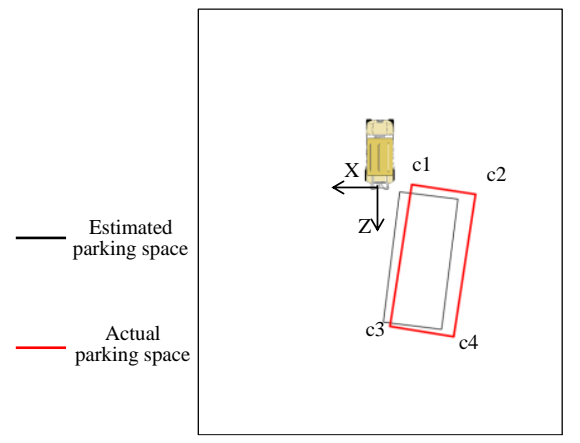

Figure 11. Estimated and actual parking space for a parking experiment at 5.8 second

\section{Conclusions}

The key issue for drivers to complete the parking mission is that they need to know the surroundings around the vehicle especially for the parking space as accurate as possible. For inexperienced drivers, it is very difficult to combine incomplete and fragmented images from side and review mirrors to establish spatial representation for the environment of the vehicle. Therefore, a parking assistance display based on stereo vision by two wide-angle cameras mounted at the rear of the vehicle is proposed to help drivers conducting parking tasks. Based on images from those two cameras, the relative position of the parking space with respect to the vehicle can be calculated. Furthermore, an inside-out display to show the top view of visual information about the parking space can therefore be drawn. Actual parking experiments with a golf cart were conducted to examine parking performance with the presented assistance display. 
Experimental results clearly demonstrate drivers are able to accomplish parallel parking tasks only with the visual information from the parking assistance display.

For future works, a number of research directions need to be deeply explored. The proposed approach has to extend to garage parking or reverse parking tasks in the near future. Obviously, a more accurate estimation algorithm for position of the parking space is required to provide consistent estimation results. In addition, the estimation algorithm for parking space has to be robust enough to overcome the difficulty caused by its corner features occluded by other objects such as adjacent vehicles.

\section{REFERENCES}

[1] Noh, B. Tae. \& Kim, S. Jae (2015) Smart Parking Assist System of Vehicle and Control Method Thereof, United States Patent 9,168,955 B2, filed March 21, 2013, and issued October 27, 2015.

[2] Jung, G. Ho, D. Kim, S. Dong \& Kim, J. (2010) "Light-stripe-projection-based target position designation for intelligent parking-assist system", IEEE Trans. Intelligent Transportation Systems, Vol. 11, No. 4, pp 942-953.

[3] Jung, G. Ho, Kim, S. Dong, Yoon, J. Pal \& Kim, J. (2008) "Two-touch type parking slot marking recognition for target parking position designation", Proc. IEEE Intelligent Vehicles Symposium, Eindhoven, The Netherlands, pp 1161-1166.

[4] Suhr, K. Jae \& Jung, G. Ho (2012) "Fully-automatic recognition of various parking slot markings in around view monitor (AVM) image sequences", Proc. IEEE Intelligent Transportation Systems, Anchorage AK, USA, pp 1294-1299.

[5] Suhr, K. Jae \& Jung, G. Ho (2014) "Sensor fusion-based vacant parking slot detection and tracking", IEEE Trans. Intelligent Transportation Systems, Vol. 15, No. 1, pp 21-36.

[6] Zhang, B., Appia, V., Pekkucuksen, I., Batur, U. Aziz, Shastry, P., Liu, S., Sivasankaran, S., Chitnis, K. \& Liu, Y. (2014) "A surround view camera solution for embedded systems", Proc. IEEE Computer Vision and Pattern Recognition Workshops (CVPRW ‘14), Columbus OH, USA, pp662-667.

[7] Wang, C., Zhang, H., Yang, M., Wang, X., Ye, L. \& Guo, C. (2014) "Automatic parking based on a bird's eye view vision system", Advances in Mechanical Engineering, Vol. 2014, Article no. 847406, 13 pages.

[8] Gojak, V., Janjatovic, J., Vukota, N., Milosevic, M., Bjelica, Z. Milan (2017) "Informational bird's eye view system for parking assistance", Proc. IEEE 7th International Conference on Consumer Electronics, Berlin, Germany, pp 103-104.

[9] Unger, C., Wahl, E. \& Ilic, S. (2014) "Parking assistance using dense motion-stereo", Machine Vision and Applications, Vol. 25, pp 561-581.

[10]Zhang, L., Li, X., Huang, J., Shan, Y. \& Wang, D. (2018) "Vision-based parking-slot detection: A benchmark and a learning-based approach", Symmetry, Vol. 10, No. 64, 18 pages.

[11]Rosten, E. \& Drummond, T. (2006) "Machine learning for high-speed corner detection", Proc. 9th European Conference on Computer Vision (ECCV '06), Lecture Notes in Computer Science, Vol. 3951. Springer, Berlin, Heidelberg, pp 430-443. 


\section{AUTHORS}

Chi-Cheng Cheng was born in Taipei, Taiwan, R.O.C. He received the B.S. and M.S. degrees in power mechanical engineering from National Tsing Hua University, Hsinchu, Taiwan, in 1981 and 1983, respectively, and the Sc.D. degree in mechanical engineering from Massachusetts Institute of Technology, Cambridge, MA, USA in 1991. He has been with National Sun Yat-Sen University in Taiwan since 1991 and is a Professor in Department of Mechanical and ElectroMechanical Engineering. He was a Visiting Scholar in the Department of Electrical and Computer Engineering of University of British Columbia, Canada in 2002 and a Visiting Professor with the School of Engineering Science in Simon Fraser

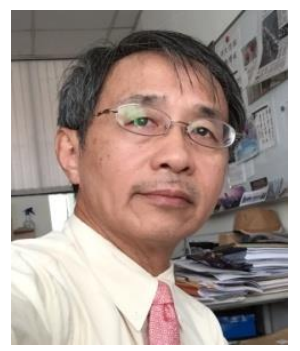
University, Canada in 2009. He was the Dean of Office of International Affairs from 2007 to 2009, the Chairman of the Department from 2014 to 2017, and Currently the Vice Dean of College of Engineering of National Sun Yat-Sen University. His research interests are in system dynamics and control, Machine vision, mechatronics, intelligent robots, and man-machine interface. Dr. Cheng has published more than 150 technical articles in refereed international journals, conferences and book chapters. He has won the Excellent Research Award from National Science Council of Taiwan in 1998, 2001, and 2002. He has been a member of Institute of Electrical and Electronics Engineers (IEEE) since 1987. He has also been a senior member of International Association of Computer Science and Information Technology (IACSIT) since 2011.

(C) 2020 By AIRCC Publishing Corporation. This article is published under the Creative Commons Attribution (CC BY) license. 